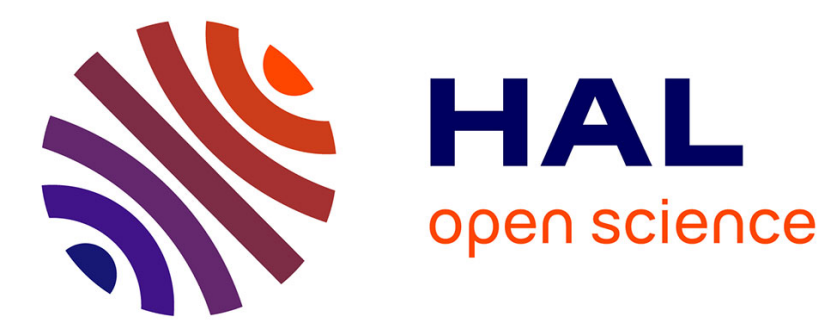

\title{
Robustness of the coherently distributed MUSIC algorithm to the imperfect knowledge of the spatial distribution of the sources
}

Wenmeng Xiong, José Picheral, Sylvie Marcos

\section{- To cite this version:}

Wenmeng Xiong, José Picheral, Sylvie Marcos. Robustness of the coherently distributed MUSIC algorithm to the imperfect knowledge of the spatial distribution of the sources. Signal, Image and Video Processing, 2017, 11 (4), pp.721-728. 10.1007/s11760-016-1015-1 . hal-01498980

HAL Id: hal-01498980

https://hal-centralesupelec.archives-ouvertes.fr/hal-01498980

Submitted on 30 Mar 2017

HAL is a multi-disciplinary open access archive for the deposit and dissemination of scientific research documents, whether they are published or not. The documents may come from teaching and research institutions in France or abroad, or from public or private research centers.
L'archive ouverte pluridisciplinaire HAL, est destinée au dépôt et à la diffusion de documents scientifiques de niveau recherche, publiés ou non, émanant des établissements d'enseignement et de recherche français ou étrangers, des laboratoires publics ou privés. 


\title{
Robustness of the coherently distributed MUSIC algorithm to the imperfect knowledge of the spatial distribution of the sources
}

\author{
Wenmeng XIONG · José PICHERAL · Sylvie MARCOS
}

the date of receipt and acceptance should be inserted later

\begin{abstract}
The MUltiple SIgnal Classification (MUSIC) estimator has been widely studied for a long time for its high resolution capabilities in the domain of the directional of arrival (DOA) estimation, with the sources assumed to be point. However, when the actual sources are spatially distributed with angular dispersion, the performance of the conventional MUSIC is degraded. This paper deals with the sensitivity of MUSIC to modeling error due to coherently distributed (CD) sources. A performance analysis of an extended MUSIC taking into account a generalized steering vector based on a CD source model (CD-MUSIC) is first studied. We establish closed-form expressions of the DOA estimation bias and mean square error due to both the model error and the effects of a finite number of snapshots. The aim of this paper is also to determine when the point source assumption is acceptable for standard MUSIC. The analytical results are validated by numerical simulations and discussed in different configurations.
\end{abstract}

Keywords array signal processing, distributed source, CD MUSIC, performance analyses

\section{Introduction}

DOA estimation issues such as the effects of model errors, the resolution of closely spaced sources, and the geometry of antennas have been widely studied in the past, with the sources assumed to be far-field point transmitters or reflectors [1-5]. Indeed, in most scenarios the point-source assumption is correct, but in some

Laboratoire des signaux et systèmes, CentraleSupélec-CNRSUniversité Paris-Sud, Université Paris Saclay

91192 Gif-sur-Yvette,France

E-mail: firstname.lastname@12s.centralesupelec.fr cases, as for instance the localization of acoustics [6] [7] or bio-medical [8] sources, ocean waves [9], or for mobile channel communication [10], a spatially distributed model of the sources is more appropriate.

The models for spatially distributed sources have been classified into two types, namely incoherently distributed (ID) sources and coherently distributed (CD) sources. On the first hand, for ID sources, signals coming from different points of the same distributed source can be considered uncorrelated, therefore the rank of the noise-free correlation matrix does not equal the number of signals [11] [12] [13]. On the other hand, in the scenario of CD sources, the received signal from different points of the same signal components are delayed and scaled replicas, therefore the rank of the noise-free correlation matrix equals the number of $\mathrm{CD}$ sources [14]. Most of the works deal with the ID model since in wireless communication, the multi-path dispersion effects are modeled as discrete ID scatterers of one source [15]. In our paper, we focus on other applications such as acoustic localization where the sources are rather modeled as continuous CD ones, this work has been partially presented in [16].

Most of the localisation methods that have been proposed to take into account the distributed sources model are based on the joint [14] [17] or iterative [12] [18] 2D estimation of the DOA and the angular dispersion parameters. These approaches present two main drawbacks: they are time consuming due to multi-parameters estimation and error modeling is introduced due to the imperfect knowledge of the angular distribution shapes of the sources. Otherwise, the standard MUSIC DOA estimator under point source hypothesis can be used despite the angular dispersion of actual sources, in this case an estimation error of the DOA arises due to the mismatch between the model and the sources. In this 
paper, we introduce an estimator called CD-MUSIC which can be considered as trade off between a $2 D$ estimator and the standard MUSIC based on the point source hypothesis. This DOA estimator exploits a generalized steering vector based on a CD source model, nevertheless it requires to know the angular distribution shape and dispersion parameters of the sources.

Previous works dealing with modeling error due to spatially distributed sources can be found in [19] for Beamforming and [20] for MUSIC. Those works are mainly based on an $1 D$ model for telecommunication applications with a small spread dispersion, and a first order Taylor approximation in the DOA estimation error is used to obtain theoretical expressions for the performances.

The motivations of the present paper are to study the sensitivity of MUSIC to error modeling due to coherently distributed sources (since the standard point source MUSIC estimator is a particular case of CDMUSIC, theoretical performance analysis provided for CD-MUSIC is also valid for standard MUSIC). Secondly, we will provide tools to determine when point source assumption is acceptable for MUSIC, when CD source model should be considered, or when more complex 2D estimators should be used. In this paper, the study is focused on applications such as acoustic imaging and source localisation [6] where due to the experiment configuration, the CD model is more appropriate, large spread dispersion is expected and hypothesis of Gaussian shape for the angular distribution is not always valid.

Modeling errors can originate from the following configurations: i) the shape (uniform, or Gaussian or others) of the angular distribution is badly known; ii) the shape of the angular spread distribution is known but with an improper spread dispersion parameter value (for example, a uniform distribution with an improper angular width, or a Gaussian distribution with an improper standard deviation, etc). To analyze the performances, we derive expressions with a second order Taylor approximation in the DOA estimation error rather than the first order techniques proposed in [19] and [20]. The result is a little more complex, but follows better the simulation especially when two sources are close and the angular dispersion parameter is over-estimated. In addition, we also consider the effect of the finite number of snapshots which was done in [21] [22] in a similar way in the scenario of point source. Here, we show that when the two problems arise simutaneously, the finite snapshots effect can be explicitly factored in the theoretical expressions for the performance analyses. Simulation results validate the theoretical results and illus- trate the limits of the point source MUSIC for a large modeling error.

The organization of this paper is as follows. The signal model and the CD-MUSIC are given in section 2 . In section 3 , the sensitivity of the estimator to the spatial dispersion of the sources is theoretically analyzed. Numerical simulations are presented in section 4 to validate the analytical expressions of the previous section. Finally, conclusions are given in section 5 .

\section{Signal model and CD-MUSIC}

\subsection{Notations}

In this paper, we use lower case boldface letters to denote vectors and upper case boldface letters to denote matrices. In addition, we use the following notations throughout the paper: $(\cdot)^{T}$ : transpose; $(\cdot)^{H}$ : Hermitian transpose; $\mathcal{R} e\{\cdot\}$ : real part; $E[\cdot]$ : expectation operator; I: identity matrix.

\subsection{Signal model}

Let us consider $q$ spatially coherently distributed farfield sources impinging on an array of $M$ sensors. The $q$ sources and $M$ signals received on the array at moment $t$ are denoted by $\mathbf{s}(t)=\left[s_{1}(t), \ldots, s_{q}(t)\right]^{T}$ and $\mathbf{y}(t)=\left[y_{1}(t), \ldots, y_{M}(t)\right]^{T}$, respectively. In the scenario of distributed sources, $\mathbf{y}(t)$ is given by:

$\mathbf{y}(t)=\mathbf{C}(\theta) \mathbf{s}(t)+\mathbf{n}(t)$

where $\mathbf{n}(t) \in \mathbb{C}^{M \times 1}$ represents additive Gaussian or uniform noise $\sigma_{b}^{2}, \mathbf{C}(\theta)=\left[\mathbf{c}_{h_{1}}\left(\theta_{1}\right), \ldots, \mathbf{c}_{h_{q}}\left(\theta_{q}\right)\right] \in \mathbb{C}^{M \times q}$ is the array steering matrix composed of $q$ steering vectors $\mathbf{c}_{h_{i}}(\theta)$ that can be written as proposed in [14]:

$\mathbf{c}_{h_{i}}\left(\theta_{i}\right)=\int_{-\frac{\pi}{2}}^{\frac{\pi}{2}} \mathbf{a}\left(\theta_{i}+\phi\right) h_{i}(\phi) d \phi$,

where $\mathbf{a}(\theta) \in \mathbb{C}^{M \times 1}$ is the steering vector for a point source which arrives from the DOA $\theta$. In the general case, $\mathbf{a}(\theta)$ can be given by $\mathbf{a}\left(\theta_{i}\right)=\left[e^{-j 2 \pi \frac{v}{\lambda} t_{1}\left(\theta_{i}\right)}, \ldots\right.$, $\left.e^{-j 2 \pi \frac{v}{\lambda} t_{m}\left(\theta_{i}\right)}, \ldots, e^{-j 2 \pi \frac{v}{\lambda} t_{M}\left(\theta_{i}\right)}\right]^{T}$, where $t_{m}\left(\theta_{i}\right)$ is the propagation time for source $i$ to the $m^{\text {th }}$ sensor, $v$ is the propagation speed of the signal and $\lambda$ is the wavelength.

The function $h_{i}(\phi)$ is introduced to describe the angular spread distribution and it can be parameterized by an angular dispersion $\Delta_{i}$ which is omitted in the notation. For instance, $\Delta_{i}$ stands for the support for a uniform distribution and for the standard deviation for a Gaussian distribution. 
The source signals and the additive noise are considered to be centered Gaussian independent random variables. Assuming that signals and noises are uncorrelated and the signal sources are uncorrelated with each other, the correlation matrix is given by: $\mathbf{R}=$ $E\left[\mathbf{y} \mathbf{y}^{H}\right]=\mathbf{C R}_{s} \mathbf{C}^{H}+\sigma_{b}^{2} \mathbf{I}$, where $\mathbf{R}_{s}$ is the source covariance matrix.

\subsection{CD-MUSIC}

Under the hypothesis that $q<M$ and $\mathbf{R}_{s}$ and $\mathbf{C}$ are not rank deficient, it is well known that the decomposition of $\mathbf{R}$ into eigenvalues $\lambda_{m}$ and eigenvectors $\mathbf{e}_{m}$ is given by: $\mathbf{R}=\sum_{m=1}^{M} \lambda_{m} \mathbf{e}_{m} \mathbf{e}_{m}^{H}=\mathbf{U} \Lambda_{s} \mathbf{U}^{H}+\sigma_{b}^{2} \mathbf{V} \mathbf{V}^{H}$, where $\mathbf{U}=\left[\mathbf{e}_{1}, \ldots, \mathbf{e}_{q}\right]$ spans the signal subspace defined by the columns of $\mathbf{C}$ and $\mathbf{V}=\left[\mathbf{e}_{q+1}, \ldots, \mathbf{e}_{M}\right]$ spans the noise subspace defined as the orthogonal complement of $\mathbf{U}, \Lambda_{s}=\operatorname{diag}\left\{\lambda_{1}, \ldots, \lambda_{q}\right\}$.

In this paper, we focus on a MUSIC based criterion which makes use of the orthogonal property of $\operatorname{span}\{\mathbf{C}\}=\operatorname{span}\{\mathbf{U}\}$ and $\operatorname{span}\{\mathbf{V}\}$ to estimate the DOA. We assume that the sources distribution shapes and the sources angular dispersion parameter $\Delta_{i}$ are known in the model, then there is only one parameter $\theta_{i}$ for the $i^{t h}$ source to estimate:

$\hat{\theta}_{i}=\underset{\theta}{\operatorname{argmax}} \frac{1}{\left\|\mathbf{c}_{h}^{H}(\theta) \mathbf{V}\right\|^{2}}$,

which is named CD-MUSIC in the following. Note that the standard point source MUSIC estimator belongs to this class of estimators, since the point source case is obtained for $\Delta=0$ and $h_{i}(\phi)$ in (2) being the Dirac delta function. In the following of this paper, the goal is to evaluate the robustness of this estimator to model errors.

\subsection{Model error definitions}

The criterion (3) can be impacted by two types of errors. First, the actual angular spread distribution $h$ of the source, may not be known in practice, in this case the function $\tilde{h}$ used by the estimator will be different from the actual $h$, that is to say, the shape of $h$ is badly known, or the shape of $h$ is known but with an error on the dispersion parameter $\Delta$. Secondly, the covariance matrix $\mathbf{R}$ is estimated from a finite number of snapshots, therefore the estimated covariance matrix $\hat{\mathbf{R}}$ and its noise subspace $\hat{\mathbf{V}}$ are different from the actual $\mathbf{R}$ and $\mathbf{V}$. Taking into consideration the two types of errors, the algorithm that we study is:

$\hat{\theta}_{i}=\arg \max _{\theta} \frac{1}{\left\|\mathbf{c}_{\tilde{h}}^{H}(\theta) \hat{\mathbf{V}}\right\|^{2}}$.
Let us introduce the definitions related to the errors. Assuming that the estimator (4) is based on an angular distribution $\tilde{h}$, the model error on the steering vector is defined as $\Delta \mathbf{c}\left(\theta_{i}\right)=\mathbf{c}_{\tilde{h}}\left(\theta_{i}\right)-\mathbf{c}_{h_{i}}\left(\theta_{i}\right)$. Similarly the model error on the covariance matrix can be defined as $\Delta \tilde{\mathbf{R}}=\tilde{\mathbf{R}}-\mathbf{R}$, where $\tilde{\mathbf{R}}=\tilde{\mathbf{C}} \mathbf{R}_{s} \tilde{\mathbf{C}}^{H}+\sigma_{b}^{2} \mathbf{I}, \tilde{\mathbf{C}}=$ $\left[\mathbf{c}_{\tilde{h}}\left(\theta_{1}\right), \ldots, \mathbf{c}_{\tilde{h}}\left(\theta_{q}\right)\right]$, and $\Delta \tilde{\mathbf{V}}=\tilde{\mathbf{V}}-\mathbf{V}$, where the subspace decomposition of $\tilde{\mathbf{R}}$ is given by $\tilde{\mathbf{R}}=\tilde{\mathbf{U}} \tilde{\Lambda}_{s} \tilde{\mathbf{U}}^{H}+$ $\sigma_{b}^{2} \tilde{\mathbf{V}} \tilde{\mathbf{V}}^{H}$. Assuming again that the covariance matrix is estimated from $N$ snapshots by the empirical estimator $\hat{\mathbf{R}}=\frac{1}{N} \sum_{n=1}^{N} \mathbf{y}\left(t_{n}\right) \mathbf{y}^{H}\left(t_{n}\right)$, the errors are defined by $\Delta \hat{\mathbf{R}}=\hat{\mathbf{R}}-\mathbf{R}$ and $\Delta \hat{\mathbf{V}}=\hat{\mathbf{V}}-\mathbf{V}$, where $\hat{\mathbf{R}}=\hat{\mathbf{U}} \hat{\Lambda}_{s} \hat{\mathbf{U}}^{H}+\sigma_{b}^{2} \hat{\mathbf{V}} \hat{\mathbf{V}}^{H}$. Let us also define $\tilde{\Pi}=\tilde{\mathbf{V}} \tilde{\mathbf{V}}^{H}$, $\hat{\Pi}=\hat{\mathbf{V}} \hat{\mathbf{V}}^{H}$, and $\Pi=\mathbf{V} \mathbf{V}^{H}$.

\section{Performance analysis}

In this section, we will investigate the effects of an imperfect knowledge of $h(\phi)$, and the finite number of snapshots on the CD-MUSIC algorithm.

\subsection{General case}

According to (4), for the $i-t h$ source, the DOA estimation $\hat{\theta}_{i}$ satisfies that the first order derivative of the denominator of (4) equals zero so that :

$2 \mathcal{R} e\left\{\dot{\mathbf{c}}_{\tilde{h}}^{H}\left(\hat{\theta}_{i}\right) \hat{\Pi} \mathbf{c}_{\tilde{h}}\left(\hat{\theta}_{i}\right)\right\}=0$,

where $\dot{\mathbf{c}}_{\tilde{h}}\left(\hat{\theta}_{i}\right)=\left.\frac{\partial \mathbf{c}(\theta)}{\partial \theta}\right|_{\hat{\theta}_{i}}$.

Assuming that, the estimation error $\left(\Delta \theta_{i}=\hat{\theta}_{i}-\theta_{i}\right)$ is small, we introduce the second order Taylor series approximations of $\mathbf{c}_{\tilde{h}}\left(\hat{\theta}_{i}\right)$ and $\dot{\mathbf{c}}_{\tilde{h}}\left(\hat{\theta}_{i}\right)$ :

$\mathbf{c}_{\tilde{h}}\left(\hat{\theta}_{i}\right) \approx \mathbf{c}_{\tilde{h}}\left(\theta_{i}\right)+\Delta \theta_{i} \dot{\mathbf{c}}_{\tilde{h}}\left(\theta_{i}\right)+\frac{1}{2} \Delta \theta_{i}^{2} \ddot{\mathbf{c}}_{\tilde{h}}\left(\theta_{i}\right)$,

$\dot{\mathbf{c}}_{\tilde{h}}\left(\hat{\theta}_{i}\right) \approx \dot{\mathbf{c}}_{\tilde{h}}\left(\theta_{i}\right)+\Delta \theta_{i} \ddot{\mathbf{c}}_{\tilde{h}}\left(\theta_{i}\right)+\frac{1}{2} \Delta \theta_{i}^{2} \dddot{\mathbf{c}}_{\tilde{h}}\left(\theta_{i}\right)$,

where $\ddot{\mathbf{c}}_{\tilde{h}}\left(\theta_{i}\right)=\left.\frac{\partial^{2} \mathbf{c}(\theta)}{\partial \theta^{2}}\right|_{\theta_{i}}$, and $\dddot{\mathbf{c}}_{\tilde{h}}\left(\theta_{i}\right)=\left.\frac{\partial^{3} \mathbf{c}(\theta)}{\partial \theta^{3}}\right|_{\theta_{i}}$.

In order to derive the expressions of the DOA estimation error, we make use of the first order approximation in [23], which says that: $\hat{\Pi}-\Pi=-\Pi \Delta \hat{\mathbf{R}} \mathbf{Q}-$ $\mathbf{Q} \Delta \hat{\mathbf{R}} \Pi$, where $\mathbf{Q}=\mathbf{U}\left(\Lambda_{s}-\sigma_{b}^{2} \mathbf{I}\right)^{-1} \mathbf{U}^{H}, \mathbf{I}$ is the $q \times q$ identity matrix.

Introducing (6) and (7) in (5), and the expression of $\hat{\Pi}$ yields:

$A\left(\theta_{i}\right) \Delta \theta_{i}^{2}+B\left(\theta_{i}\right) \Delta \theta_{i}+C\left(\theta_{i}\right)=0$,

where the terms of order greater than 2 in $\Delta \theta_{i}$ have been neglected, and the scalar $A\left(\theta_{i}\right), B\left(\theta_{i}\right), C\left(\theta_{i}\right)$ are defined below with $B\left(\theta_{i}\right)=B_{1}\left(\theta_{i}\right)+B_{2}\left(\theta_{i}\right)$ and $C\left(\theta_{i}\right)=$ 
$C_{1}\left(\theta_{i}\right)+C_{2}\left(\theta_{i}\right)$. In the following, we omit the $\theta_{i}$ in the notations for simplicity:

$$
\begin{aligned}
A & =\mathcal{R} e\left\{\frac{1}{2} \dot{\mathbf{c}}_{\tilde{h}}^{H} \Pi \ddot{\mathbf{c}}_{\tilde{h}}+\ddot{\mathbf{c}}_{\tilde{h}}^{H} \Pi \dot{\mathbf{c}}_{\tilde{h}}+\frac{1}{2} \dddot{\mathbf{c}}_{\tilde{h}}^{H} \Pi \mathbf{c}_{\tilde{h}}\right\}, \\
B_{1} & =\mathcal{R} e\left\{\dot{\mathbf{c}}_{\tilde{h}}^{H} \Pi \dot{\mathbf{c}}_{\tilde{h}}+\ddot{\mathbf{c}}_{\tilde{h}}^{H} \Pi \mathbf{c}_{\tilde{h}}\right\}, \\
B_{2} & =\mathcal{R} e\left\{2 \dot{\mathbf{c}}_{\tilde{h}}^{H} \Pi \Delta \hat{\mathbf{R}} \mathbf{Q} \dot{\mathbf{c}}_{\tilde{h}}+\ddot{\mathbf{c}}_{\tilde{h}}^{H} \Pi \Delta \hat{\mathbf{R}} \mathbf{Q} \mathbf{c}_{\tilde{h}}\right\}, \\
C_{1} & =\mathcal{R} e\left\{\dot{\mathbf{c}}_{\tilde{h}}^{H} \Pi \mathbf{c}_{\tilde{h}}\right\}, \quad C_{2}=\mathcal{R} e\left\{\dot{\mathbf{c}}_{\tilde{h}}^{H} \Pi \Delta \hat{\mathbf{R}} \mathbf{Q} \mathbf{c}_{\tilde{h}}\right\} .
\end{aligned}
$$

The expression of $\Delta \theta_{i}$ can be obtained by solving the $2^{\text {nd }}$ order equation (8).The CD-MUSIC criterion chooses the minimum value of the denominator as the estimation result, so $\hat{\theta}_{i}$ should satisfy: $\left.\frac{\partial^{2} \mathbf{c}_{\tilde{h}}^{H}(\theta) \hat{\Pi} \mathbf{c}_{\tilde{h}}(\theta)}{\partial \theta^{2}}\right|_{\hat{\theta}_{i}}>$ 0 , which makes it possible to choose the solution of $(8)$ :

$\Delta \theta_{i}=\frac{-B+\sqrt{B^{2}-4 A C}}{2 A}$.

It follows that the estimation bias and the MSE are given by:

$E\left[\Delta \theta_{i}\right]=\frac{-B_{1}+\sqrt{B_{1}^{2}-4 A C_{1}}}{2 A}$,

$E\left[\Delta \theta_{i}^{2}\right]=\frac{B_{1}^{2}-2 A C_{1}-B_{1} \sqrt{B_{1}^{2}-4 A C_{1}}}{2 A^{2}}+$

$\frac{\sigma_{b}^{2}}{N}\left[\left(\frac{1}{4 A^{2}}-\frac{B_{1}}{4 A^{2} \sqrt{B_{1}^{2}-4 A C_{1}}}\right) \varphi+\frac{\chi}{2 A \sqrt{B_{1}^{2}-4 A C_{1}}}\right]$

The calculations and the definitions of $\varphi$ and $\chi$ can be found in Appendix A.

It is interesting to see that the MSE is composed of two terms: one only depends on the model error, the other depends on the model error but with a factor $\sigma_{b}^{2} / N$. It can be expected that the second term will be negligible when $N$ increases.

\subsection{First order approximation}

In this subsection, we discuss the situation where the estimation error $\Delta \theta_{i}$ is small enough, so that the second order terms in $\Delta \theta_{i}$ can be negligible with respect to the first order terms. Keeping only the first order terms in (8) yields:

$B \Delta \theta_{i}+C=0$.

Introducing the property $\Pi \mathbf{c}_{\tilde{h}}=\Pi \Delta \mathbf{c}$, and neglecting the second order terms in $\Delta \theta_{i} \Delta \mathbf{c}$ and $\Delta \theta_{i} \Delta \hat{\mathbf{R}},(12)$ becomes:

$\Delta \theta_{i} \dot{\mathbf{c}}_{\tilde{h}}^{H} \Pi \dot{\mathbf{c}}_{\tilde{h}}=\mathcal{R} e\left\{\dot{\mathbf{c}}_{\tilde{h}}^{H} \Pi \Delta \mathbf{c}+\dot{\mathbf{c}}_{\tilde{h}}^{H} \Pi \Delta \hat{\mathbf{R}} \mathbf{Q} \mathbf{c}_{\tilde{h}}\right\}$
Replacing $\Pi$ by $\tilde{\Pi}-\Delta \tilde{\Pi}$, and neglecting the second order terms in $\Delta \tilde{\Pi} \Delta \theta_{i}, \Delta \tilde{\Pi} \Delta \mathbf{c}$ and $\Delta \tilde{\Pi} \Delta \hat{\mathbf{R}}$, the expression of the estimation error of DOA can be simplified as:

$\Delta \theta_{i}=\frac{\mathcal{R} e\left\{\dot{\mathbf{c}}_{\tilde{h}}^{H} \tilde{\Pi} \Delta \mathbf{c}\right\}}{\dot{\mathbf{c}}_{\tilde{h}}^{H} \tilde{\Pi} \dot{\mathbf{c}}_{\tilde{h}}}+\frac{\mathcal{R} e\left\{\dot{\mathbf{c}}_{\tilde{h}}^{H} \tilde{\Pi} \Delta \hat{\mathbf{R}} \mathbf{Q} \mathbf{c}_{\tilde{h}}\right\}}{\dot{\mathbf{c}}_{\tilde{h}}^{H} \tilde{\Pi} \dot{\mathbf{c}}_{\tilde{h}}}$.

As $\Delta \hat{\mathbf{R}}$ follows a Wishart distribution, $E[\Delta \hat{\mathbf{R}}]=0$ [24], it follows that the estimation bias is derived as:

$E\left[\Delta \theta_{i}\right]=\frac{\mathcal{R} e\left\{\dot{\mathbf{c}}_{\tilde{h}}^{H} \tilde{\Pi} \Delta \mathbf{c}\right\}}{\dot{\mathbf{c}}_{\tilde{h}}^{H} \tilde{\Pi} \dot{\mathbf{c}}_{\tilde{h}}}$,

and the MSE can be given by:

$E\left[\Delta \theta_{i}^{2}\right]=\left(\frac{\mathcal{R} e\left\{\dot{\mathbf{c}}_{\tilde{h}}^{H} \tilde{\Pi} \Delta \mathbf{c}\right\}}{\dot{\mathbf{c}}_{\tilde{h}}^{H} \tilde{\Pi} \dot{\mathbf{c}}_{\tilde{h}}}\right)^{2}+\frac{\sigma_{b}^{2}}{2 N} \cdot \frac{\mathcal{R} e\left\{\dot{\mathbf{c}}_{\tilde{h}}^{H} \tilde{\Pi} \dot{\mathbf{c}}_{\tilde{h}} \mathbf{c}_{\tilde{h}}^{H} \mathbf{Q R Q} \mathbf{c}_{\tilde{h}}\right\}}{\left(\dot{\mathbf{c}}_{\tilde{h}}^{H} \tilde{\Pi} \dot{\mathbf{c}}_{\tilde{h}}\right)^{2}}$

Note that this first order approximation makes it possible to express (15) and the first term of (16) as an explicit function of the model error $\Delta \mathbf{c}$.

To obtain (15) and (16), the second order of Talyor approximation in $\Delta \theta_{i}$ has been ignored. Therefore, (15) and (16) are valid in the case that $\Delta \theta_{i}$ is small enough, while (10) and (11) are also valid for a larger $\Delta \theta_{i}$.

Assuming that the source angular distribution is symmetric and the source dispersion is small enough, and the array aperture is not too large, we introduce a second order Taylor approximation in $\phi$ such that:

$\mathbf{c}_{h_{i}}\left(\theta_{i}\right)=\int_{-\frac{\pi}{2}}^{\frac{\pi}{2}} \mathbf{a}\left(\theta_{i}+\phi\right) h_{i}(\phi) d \phi \approx \mathbf{a}\left(\theta_{i}\right)+\frac{1}{2} \ddot{\mathbf{a}}\left(\theta_{i}\right) \sigma_{h}^{2}$,

$\mathbf{c}_{\tilde{h}_{i}}\left(\theta_{i}\right)=\int_{-\frac{\pi}{2}}^{\frac{\pi}{2}} \mathbf{a}\left(\theta_{i}+\phi\right) \tilde{h}_{i}(\phi) d \phi \approx \mathbf{a}\left(\theta_{i}\right)+\frac{1}{2} \ddot{\mathbf{a}}\left(\theta_{i}\right) \sigma_{\tilde{h}}^{2}$,

where $\sigma_{h}^{2}=\int \phi^{2} h(\phi) d \phi, \sigma_{\tilde{h}}^{2}=\int \phi^{2} \tilde{h}(\phi) d \phi$ is the angular spread of the source and the model, respectively.

For the sake of simplicity, assume again that we are in the case of one source. Taking into account that $\tilde{\Pi}=$ $\mathbf{I}-\mathbf{c}_{\tilde{h}}\left(\mathbf{c}_{\tilde{h}}^{H} \mathbf{c}_{\tilde{h}}\right)^{-1} \mathbf{c}_{\tilde{h}}^{H}$, and introducing (17) and (18) in (15), the estimation bias can be given by:

$E\left[\Delta \theta_{i}\right]=\frac{1}{2} \frac{\left(\sigma_{\tilde{h}}^{2}-\sigma_{h}^{2}\right)\left(\mathbf{a}^{H} \ddot{\mathbf{a}} \dot{\mathbf{a}}^{H} \mathbf{a}-M \dot{\mathbf{a}}^{H} \ddot{\mathbf{a}}\right)}{M \dot{\mathbf{a}}^{H} \dot{\mathbf{a}}-\dot{\mathbf{a}}^{H} \mathbf{a} \mathbf{a}^{H} \dot{\mathbf{a}}}$,

where $\mathbf{a}$ is simplified for $\mathbf{a}\left(\theta_{i}\right), \dot{\mathbf{a}}=\frac{\partial \mathbf{a}}{\partial \theta}, \ddot{\mathbf{a}}=\frac{\partial^{2} \mathbf{a}}{\partial \theta^{2}}, \dddot{\mathbf{a}}=$ $\frac{\partial^{3} \mathbf{a}}{\partial \theta^{3}}$. 
Let us recall that $\mathbf{a}\left(\theta_{i}\right)=\left[e^{-j \tau_{1}}, \ldots, e^{-j \tau_{m}}, \ldots, e^{-j \tau_{M}}\right]$, where $\tau_{m}=2 \pi \frac{v}{\lambda} t_{m}\left(\theta_{i}\right)$. The estimation bias can be given as:

$$
\begin{aligned}
& E\left[\Delta \theta_{i}\right] \\
& =\frac{1}{2} \frac{\left(\sigma_{\tilde{h}}^{2}-\sigma_{h}^{2}\right)\left(\sum_{m=1}^{M} \ddot{\tau}_{m} \sum_{m=1}^{M} \dot{\tau}_{m}-M \sum_{m=1}^{M} \dot{\tau}_{m} \ddot{\tau}_{m}\right)}{M \sum_{m=1}^{M} \dot{\tau}_{m}^{2}-\left(\sum_{m=1}^{M} \dot{\tau}_{m}\right)^{2}}
\end{aligned}
$$

where $\dot{\tau}_{m}=\frac{\partial \tau_{m}}{\partial \theta}, \ddot{\tau}_{m}=\frac{\partial^{2} \tau_{m}}{\partial \theta^{2}}$.

An important result is that, at first order, the estimation bias only depends on the difference of the angular spread of the source and the model and not on their shapes.

Assuming a linear array is used, $\tau_{m}=2 \pi d_{m} \sin (\theta)$, where $d_{m}$ is the distance between the $m^{\text {th }}$ sensor and the origin, and the estimation bias can be given as:

$E\left[\Delta \theta_{i}\right]=\frac{1}{2}\left(\sigma_{\tilde{h}}^{2}-\sigma_{h}^{2}\right) \tan (\theta)$.

From (19) and (22) we can see that the sign of the estimation bias depends on the difference between the angular spread of the source and the model. Note that in the case of a uniform distribution of the spatial dispersion, $\sigma_{h}^{2}=\Delta^{2} / 12$ while for the Gaussian distribution $\sigma_{h}^{2}=\Delta^{2}$.

\section{Numerical results}

In this section, numerical examples are presented to illustrate the validity of the analytical results of the estimation performances established in section 3 . In all simulations, source signals $\mathbf{s}(t)$ and noise $\mathbf{n}(t)$ are complex Gaussian independent random variables, with zero as mean value and 1 as source power. A uniform linear array (ULA) is composed of $M=10$ omni-directional sensors spaced by half-wavelength. In this case the steering vector pour point source $i$ can be given as $\mathbf{a}\left(\theta_{i}\right)=$ $\left[1, e^{-j \pi \sin \left(\theta_{i}\right)}, \ldots, e^{-j \pi(M-1) \sin \left(\theta_{i}\right)}\right]^{T}$. The steering vector for uniformly distributed and Gaussian source can be given as $\mathbf{c}_{h_{i}}\left(\theta_{i}\right)=\frac{1}{\Delta} \int_{-\frac{\Delta}{2}}^{\frac{\Delta}{2}} \mathbf{a}\left(\theta_{i}+\phi\right) d \phi$, and $\mathbf{c}_{h_{i}}\left(\theta_{i}\right)=$ $\frac{1}{\sqrt{2 \pi} \Delta} \int_{-\infty}^{+\infty} \mathbf{a}\left(\theta_{i}+\phi\right) \exp \left\{-\frac{\phi^{2}}{2 \Delta^{2}}\right\} d \phi$, respectively. In addition, $S N R=10 d B$, and $N=1000$ snapshots. Each Monte-Carlo simulation is a realization of source signals and additive noise. Different analytical results are compared to simulation results. We first present the validation of our theoretical results and then we analyze the performance.

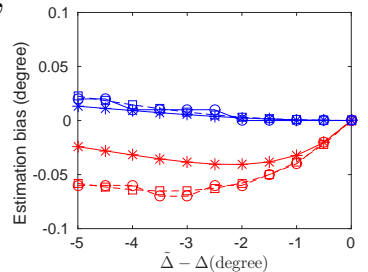

(a) $\tilde{\Delta}<\Delta$

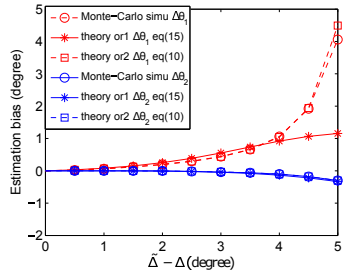

(b) $\tilde{\Delta}>\Delta$
Fig. 1 Estimation bias vs. the angular dispersion model error. (2 sources)

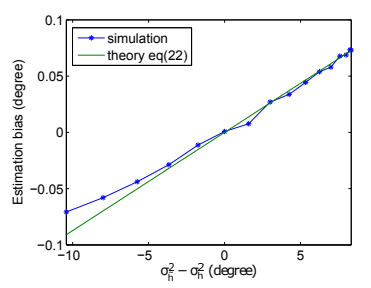

(a) Uniform source and rectangular model

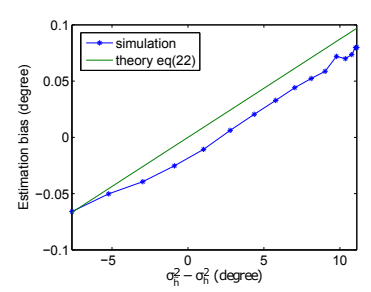

(b) Uniform source and Gaussian model
Fig. 2 DOA estimation error vs. difference of the angular spread between signal and model. (1 source)

\subsection{Validation of theoretical results}

The validation of estimation bias formulas (10) and (15) is illustrated in figure 1 , with two uniform distributed sources $\left(\theta_{1}=33^{\circ}, \theta_{2}=47^{\circ}\right)$, and with a same angular dispersion $\Delta=10^{\circ}$. Figure 1 (a) shows the error for an underestimation of the dispersion parameter $\Delta$ while Figure 1(b) shows error for an overestimation. As expected, the estimation bias is null when the exact model parameter $\tilde{\Delta}=\Delta=10^{\circ}$ is used. Comparing the simulation results, one can observe that the DOA estimation bias is smaller when the parameter $\tilde{\Delta}$ is smaller than $\Delta$ (Figure 1(a)), than when it is larger than $\Delta$ (Figure $1(\mathrm{~b})$ ). Focusing on the validity of the expressions derived in the previous section, we can notice that for both $\theta_{1}$ and $\theta_{2}$, the DOA estimation bias obtained in (10) outperforms the one obtained in (15), where the advantage of (10) is much more evident for $\theta_{1}$, which has a larger estimation error.

Figure 2 illustrates the validation of the results obtained in (22) as a function of the difference of the angular spread of the source and model, in order to express the estimation error as an explicit function of the model error. In this scenario one source is considered at $\theta_{0}=45^{\circ}$ with uniform distribution and $\Delta$ varies from $0^{\circ}$ to $15^{\circ}$, the model is uniform in figure 2(a) and Gaussian in figure 2(b). The origin is placed at the center of the array. We can see that for such an array configu- 


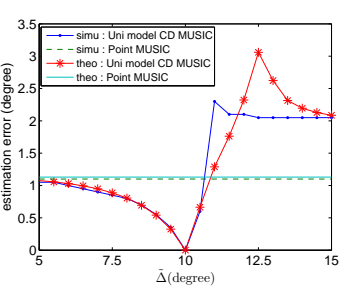

(a) Uniform distribution (b) Gaussian distribution and point source models and point source models

Fig. 3 DOA estimation error $\left|\Delta \theta_{2}\right|$ vs. angular dispersion of the model (2 sources)

ration, the sign of the estimation bias depends on the difference between angular dispersion of the source and the model. In particular, when the angular spread of the source equals to the angular spread of the model, the estimation bias vanishes.

\subsection{Robustness to shape mismatch}

These simulations investigate the impact of the model error due to a mismatch of the shape of the angular distribution. To localize a source with an actual uniform distribution, we compare the performance of three estimators: i) MUSIC, assuming a point source model (figure 3(a), 3(b)), ii) CD-MUSIC assuming a uniform angular dispersion (figure 3(a)), or iii) a Gaussian angular dispersion (figure 3(b)). We consider the case where two uniform distributed sources arrive from $\theta_{1}=28^{\circ}$ and $\theta_{2}=32^{\circ}$, with an angular dispersion $\Delta=10^{\circ}$, assuming that the covariance matrix is known. Estimation error from simulation and theoretical equations (10) are plotted versus the model dispersion parameter $\tilde{\Delta}$.

In figure 3(a), it is shown that the model error due to a point source model (point model MUSIC) provides a larger estimation error than the model error due to a mismatch on parameter $\Delta$, except when $\Delta$ is overestimated.

As expected, the error due to distributed sources can be canceled with CD-MUSIC if the source dispersion parameters are known (in this case : uniform distribution and $\tilde{\Delta}=\Delta=10^{\circ}$ ). Moreover, if the dispersion is only roughly known (i.e. $\tilde{\Delta} \approx \Delta=10^{\circ}$ one can see that CD-MUSIC still outperforms standard MUSIC unless dispersion is too overestimated.

When the model error is due to the use of a Gaussian shape instead of a uniform one (figure 3(b)), we can note that the CD-MUSIC error can be also reduced near to zero for the value $\tilde{\Delta} \approx 3.5^{\circ}$, which approximately corresponds to the case $\sigma_{h}^{2}=\sigma_{\tilde{h}}^{2}$, when $\Delta=10^{\circ}$ is the

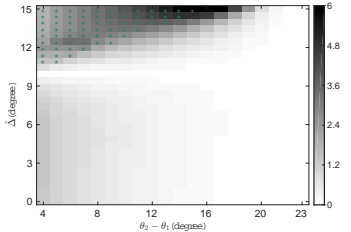

(a) Simulation

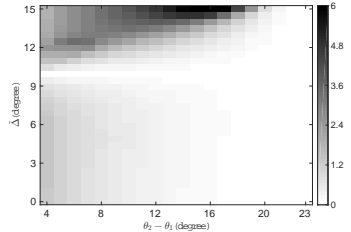

(b) Theory eq. (10)
Fig. $4\left|E\left[\Delta \theta_{1}\right]\right|$ vs. the source angular separation $\left|\theta_{2}-\theta_{1}\right|$ and model angular dispersion $\tilde{\Delta}$ (2 sources)

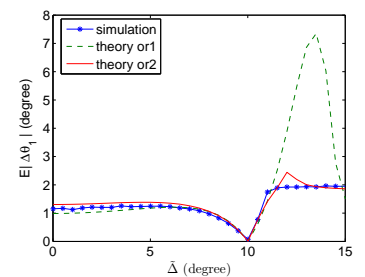

(a) Close sources case

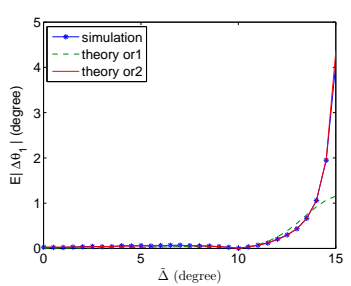

(b) Separated sources case
Fig. $5\left|E\left[\Delta \theta_{1}\right]\right|$ vs. model angular dispersion $\tilde{\Delta}$ (2 sources)

spread of the rectangular model and $\Delta=3.5^{\circ}$ is the standard deviation of the Gaussian model.

The results reveal the robustness of CD-MUSIC to the spatial distribution of the sources. Even if we take an angular distribution shape for the model which is different from that of the source signal, it is possible to have a DOA estimation bias near to zero providing $\tilde{\Delta}$ is well chosen. The only case where standard MUSIC outperforms CD-MUSIC is when the dispersion is badly known and it is overestimated with respect to the actual value. Also note that in this scenario the theoretical expression fits the simulation results except when resolution problems arise (mainly when the parameter $\Delta$ is over-estimated).

\subsection{Angular separation}

In order to explore the resolution capabilities of CDMUSIC in presence of model errors, figure 4 shows the absolute value of the DOA estimation bias when the model error due to the dispersion parameter $(\tilde{\Delta})$ and the angle between the two sources $\left(\theta_{2}-\theta_{1}\right)$ both vary. In this scenario, we consider two sources with uniform angular dispersion $\left(\Delta=10^{\circ}\right)$ and with $\theta_{m}=\frac{1}{2}\left(\theta_{1}+\right.$ $\left.\theta_{2}\right)=30^{\circ}$. The stars mark the region where the sources are not resolved, that is to say, the two sources are so close that the CD-MUSIC criterion has only one peak in the middle. In order to highlight the behavior of the estimator, figure 5 plots the estimation error in two extreme cases : for close sources $\left(\theta_{2}-\theta_{1}=4^{\circ}\right.$ - figure 


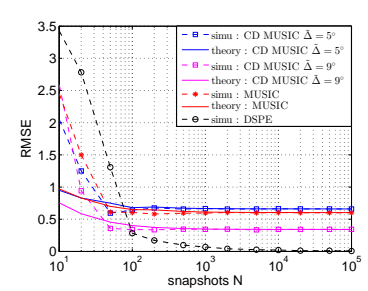

(a) source $n^{\circ} 1\left(\theta_{1}=26^{\circ}\right)$

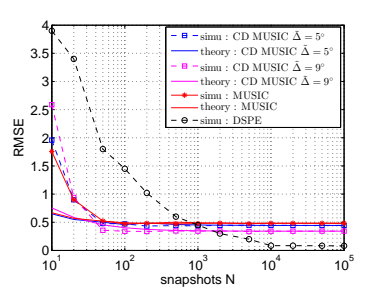

(b) source $n^{\circ} 2\left(\theta_{2}=34^{\circ}\right)$
Fig. 6 DOA RMSE vs. number of snapshots (2 sources) 100 Monte-Carlo simulations)

$5(\mathrm{a}))$ and for separated sources $\left(\theta_{2}-\theta_{1}=18^{\circ}\right.$ - figure $5(\mathrm{~b}))$.

From these results one can observe that firstly, CDMUSIC achieves high resolution performance for CD sources under the constraint of small modeling error (i.e. $\tilde{\Delta} \approx \Delta=10^{\circ}$ ). In contrary, standard point source MUSIC estimator (i.e. $\tilde{\Delta}=0$ ) can not provide reliable estimate for close CD sources. Secondly, when CD sources are well separated, CD-MUSIC is robust to model error on the angular dispersion parameter unless it is overestimated $(\tilde{\Delta}>\Delta)$. Focussing on point source $\operatorname{MUSIC}(\Delta)$, one can notice that it provides accurate estimates in this case. In conclusion, unless for high resolution purpose, error modeling due to CD sources can be neglected.

\subsection{Performance versus the number of snapshots}

In figure 6, the RMSE (root mean square error) of the estimator is plotted versus the snapshots number. A uniform source and a Gaussian source arrive from $\theta_{1}=26^{\circ}$ and $\theta_{2}=34^{\circ}$, respectively. We compare the performance of CD-MUSIC (with uniform distributed model in two cases: with a large error on dispersion parameter $\tilde{\Delta}=5^{\circ}$ and with a smaller error $\tilde{\Delta}=9^{\circ}$ ), MUSIC for point source, and DSPE (with uniform source model). DSPE proposed in [14] estimates jointly the DOA and the source angular dispersion parameter, using a $2 D$ MUSIC based approach but it is more timeconsuming. The RMSE for CD-MUSIC and MUSIC decrease as well as $N$ increases, and then converge to non zero values given by (16). This reveals that when there are two perturbations, the finite number of snapshots effect dominates in the case of a small number of snapshots whereas the model error effect dominates when the number of snapshots is large. Also, in the case where the finite number of snapshots effect dominates, the $1 D$ estimators have better performances; in contrary, when the model error effect dominates, the $2 D$ estimator has better performance.
The advantage of CD-MUSIC in the case that the information of angular distribution of the actual sources is roughly known is illustrated by these simulations. When the model error is small (case $\tilde{\Delta}=9^{\circ}$ ) CDMUSIC outperforms conventional MUSIC but also the more complex estimator DSPE for $N<100$ or $N<$ 1000 according to the type of sources. When the model error is larger (case $\tilde{\Delta}=5^{\circ}$ ) CD-MUSIC is almost equivalent to MUSIC except for a small number of snapshots.

Note that for CD-MUSIC and MUSIC, when $N$ is too small, the theoretical results given by (16) do not suit the simulation results, because a first order of approximation of $\Delta \hat{\mathbf{R}}$ is not enough when $\Delta \hat{\mathbf{R}}$ is too large.

\section{Conclusion}

This paper has investigated the effects of both the angular dispersion of the source and the finite number of snapshots on the behavior of the CD-MUSIC. New analytical expressions of the DOA estimation bias and MSE as a function of these two perturbations have been given based on the second order Taylor approximation in order to provide accurate expressions even for a large error.

Numerical simulations are in adequacy with the proposed theoretical results. We have shown that, CDMUSIC can be viewed as a trade-off between the standard MUSIC and 2D estimator DSPE. In the case that the angular dispersions of the sources are roughly known, it outperforms the standard MUSIC and the performance can be quite similar to DSPE (or even better for a small number of samples) while DSPE has the main drawback to require the maximization of a $2 D$ criterion. We also have shown that, at the first order and in the one source case, the DOA estimation bias only depends on the difference of the angular spreads between the model and signal spatial distributions and not on their shapes. These results also make it possible to determine when we can use the standard MUSIC and which error shall be expected, and when, on the contrary, it will be necessary to use a more computationally complex $2 D$ method such as DSPE.

\section{Appendix A}

Based on (9), we study the case that the number of snapshots $N$ is big enough, so that $\Delta \hat{\mathbf{R}}, B_{2}, C_{2}$ is small, we can make the approximation :

$\sqrt{B^{2}-4 A C} \approx \sqrt{B_{1}^{2}-4 A C_{1}}\left(1+\frac{1}{2} \frac{2 B_{1} B_{2}+B_{2}^{2}-4 A C_{2}}{B_{1}^{2}-4 A C_{1}}\right)$. 
Introducing (23) in (9), and keeping only first order terms in $\Delta \hat{\mathbf{R}}$, the expression of DOA estimation error can be given by:

$\Delta \theta_{i} \approx \frac{-\left(B_{1}+B_{2}\right)+\sqrt{B_{1}^{2}-4 A C_{1}}\left(1+\frac{1}{2} \frac{2 B_{1} B_{2}-4 A C_{2}}{B_{1}^{2}-4 A C_{1}}\right)}{2 A}$.

$\Delta \hat{\mathbf{R}}$ is a Wishart distribution [24] matrix with the property $E[\Delta \hat{\mathbf{R}}]=0$, so that $E\left[B_{2}\right]=E\left[C_{2}\right]=0$. It follows that the DOA estimation bias can be derived as (10). Similarly, the DOA estimation MSE can be given by:

$E\left[\Delta \theta_{i}^{2}\right]=\frac{B_{1}^{2}-2 A C_{1}-B_{1} \sqrt{B_{1}^{2}-4 A C_{1}}}{2 A^{2}}$
$+\left(1 / 2 A^{2}-\frac{B_{1}}{2 A^{2} \sqrt{B_{1}^{2}-4 A C_{1}}}\right) E\left[B_{2}^{2}\right]+\frac{1}{A \sqrt{B_{1}^{2}-4 A C_{1}}} E\left[B_{2} C_{2}\right]$.

Using the same method in [21], we obtain $E\left[B_{2}^{2}\right]=\frac{\sigma_{b}^{2}}{2 N} \varphi$ and $E\left[B_{2} C_{2}\right]=\frac{\sigma_{b}^{2}}{2 N} \chi$, where:

$$
\begin{aligned}
& \varphi \triangleq \mathcal{R} e\left\{4 \dot{\mathbf{c}}_{h}\left(\theta_{i}\right)^{H} \Pi \dot{\mathbf{c}}_{h}\left(\theta_{i}\right) \dot{\mathbf{c}}_{h}\left(\theta_{i}\right)^{H} \mathbf{Q R Q} \dot{\mathbf{c}}_{h}\left(\theta_{i}\right)\right. \\
& +4 \ddot{\mathbf{c}}_{h}\left(\theta_{i}\right)^{H} \Pi \dot{\mathbf{c}}_{h}\left(\theta_{i}\right) \mathbf{c}_{h}\left(\theta_{i}\right) \mathbf{Q R Q c _ { h }}\left(\theta_{i}\right) \\
& \left.+\ddot{\mathbf{c}}_{h}\left(\theta_{i}\right)^{H} \Pi \ddot{\mathbf{c}}_{h}\left(\theta_{i}\right) \mathbf{c}_{h}\left(\theta_{i}\right) \mathbf{Q R Q} \mathbf{c}_{h}\left(\theta_{i}\right)\right\} \text {, } \\
& \chi \triangleq \mathcal{R} e\left\{2 \dot{\mathbf{c}}_{h}\left(\theta_{i}\right)^{H} \Pi \dot{\mathbf{c}}_{h}\left(\theta_{i}\right) \dot{\mathbf{c}}_{h}\left(\theta_{i}\right) \mathbf{Q R Q} \mathbf{c}_{h}\left(\theta_{i}\right)\right. \\
& \left.+\ddot{\mathbf{c}}_{h}\left(\theta_{i}\right)^{H} \Pi \dot{\mathbf{c}}_{h}\left(\theta_{i}\right) \mathbf{c}_{h}\left(\theta_{i}\right) \mathbf{Q R Q} \mathbf{c}_{h}\left(\theta_{i}\right)\right\} .
\end{aligned}
$$

\section{References}

1. Domenico Ciuonzo, Gianmarco Romano, and Raffaele Solimene. Performance analysis of time-reversal MUSIC. IEEE Transactions on Signal Processing, 63(10):26502662, 2015.

2. Guang-wei Ma, Zhi-chao Sha, Zhang-meng Liu, and ZhiTao Huang. Ica-based direction-of-arrival estimation of uncorrelated and coherent signals with uniform linear array. Signal, Image and Video Processing, 8(3):543-548, 2014.

3. Chunjing Liu, Feng Liu, and Shu Zhang. A novel direction-of-arrival estimation method for wideband signals. Signal, Image and Video Processing, 6(1):141-145, 2012.

4. Jian-Wu Tao, Wen-Xiu Chang, and Wei Cui. Directionfinding of coherent signals based on cylindrical vectorhydrophones array. Signal, Image and Video Processing, 4(2):221-232, 2010.

5. H El Markhi, MMO Haibala, F Mrabti, Pascal Chargé, and M Zouak. An improved cyclic beamforming method for signal doa estimation. Signal, Image and Video Processing, 1(3):267-272, 2007.

6. Ning Chu, Ali Mohammad Djafari, and José Picheral. Robust Bayesian super-resolution approach via sparsity enforcing a priori for near-field aeroacoustic source imaging. Journal of Sound and Vibration, 332(18):4369-4389, Fev. 2013.

7. T.F.Brooks and W.M.Humphreys. A deconvolution approach for the mapping of acoustic source(DAMAS) determined from phased microphone arrays. Journal of Sound and Vibration, 294():856-879, July 2006.

8. Gwénaël Birot, Laurent Albera, Fabrice Wendling, and Isabelle Merlet. Localisation of extended brain sources from EEG/MEG: the ExSo-MUSIC approach. Elsiever Neuroimage, 56(1):102-113, May 2011.
9. Adam P Young, RT Guza, Peter N Adams, William C O'Reilly, and Reinhard E Flick. Cross-shore decay of cliff top ground motions driven by local ocean swell and infragravity waves. Journal of Geophysical Research: Oceans (1978-2012), 117(C6), 2012.

10. K.I.Pedersen, P.E.Mogensen, and B.H.Fleury. A stochastic model of the temporal and azimuthal dispersion seen at the base station in outdoor propagation environments. IEEE Transaction on Vehicular Technology, 49(2), Mar. 2000.

11. O.Besson and P.Stoica. Decoupled estimation of DOA and angular spread of a spatially distributed source. IEEE Transaction on Signal Processing, 48(7):1872-1882, July 2000.

12. A.Zoubir, Y.Wang, and Pascal Chargé. Efficient subspace-based estimator for localization of multiple incoherently distributed sources. IEEE Transaction on Signal Processing, 56(2):532-542, Feb. 2008.

13. A.Hu, T.Lv, H.Gao, Z.Zhang, and S.Yang. An ESPRITbased approach for 2-d localization of incoherently distibuted sources in massive MIMO sYSTEMS. IEEE Journal of Selected Topics in Signal processing, 2014.

14. S.Valaee, B.Champagne, and P.Kabal. Parametric localization of distributed sources. IEEE Transactions on Signal Processing, 43(9):2144-2153, Sept. 1995.

15. Raviv Raich, Jason Goldberg, and Hagit Messer. Bearing estimation for a distributed source: Modeling, inherent accuracy limitations and algorithms. Signal Processing, IEEE Transactions on, 48(2):429-441, Feb. 2000.

16. Wenmeng Xiong, Jose Picheral, and Sylvie Marcos. Performance analysis of MUSIC in the presence of modeling errors due to the spatial distributions of sources. In Acoustics, Speech and Signal Processing (ICASSP), 2015 IEEE International Conference on, pages 2804-2808. IEEE, 2015.

17. Tonu Trump and Björn Ottersten. Estimation of nominal direction of arrival and angular spread using an array of sensors. Signal Processing, 50(12):57-69, Apr. 1996.

18. J.Lee, I.Song, H.Kwon, and S.Lee. Low-complexity estimation of 2D DOA for coherently distributed sources. Signal Processing, 83(8):1789-1802, 2003.

19. Raviv Raich, Jason Goldberg, and H Messer. Bearing estimation for a distributed source via the conventional beamformer. In Statistical Signal and Array Processing, 1998. Proceedings., Ninth IEEE SP Workshop on, pages 58. IEEE, 1998

20. David Astély and Bjorn Ottersten. The effects of local scattering on direction of arrival estimation with MUSIC. Signal Processing, IEEE Transactions on, 47(12):32203234, Dec. 1999.

21. A.Swindlehurst and T.Kailath. A performance analysis of subspace method in the presence of model errors : Case of MUSIC. IEEE Transactions on Signal Processing, 40(7):1758-1773, July 1992.

22. A.Kangas, P.Stoica, and T.Soderstrom. Finite sample and modeling error effects on ESPRIT and MUSIC diretion estimators. Radar, Sonar and Navigation, IEE Proceedings, 141(5):249-255, Oct. 1994.

23. A.J.Weiss and B.Friedlander. Effects of modeling errors on the resolution threshold of the MUSIC algorithm. IEEE Transactions on Signal Processing, 42(6):1519-1526, June 1994.

24. John A Tague and Curtis I Caldwell. Expectations of useful complex wishart forms. Multidimensional Systems and Signal Processing, 5(3):263-279, 1994. 